\title{
Sometimes it is not what it seems: Animal-type melanoma, an unusual pigmented lesion of the scalp
}

\author{
Barreiro $D^{1 *}$, Costa $C^{2,3}$, Gullo $\mathbf{I}^{2,3,4}$, Menes $\mathrm{M}^{1}$, Oliveira $\mathrm{O}^{1}$ and Silva $\mathrm{A}^{1}$ \\ ${ }^{1}$ Department of Plastic Surgery and Burn Unit, Centro Hospitalar São João, Porto, Portugal \\ ${ }^{2}$ Department of Pathology, Centro Hospitalar São João, Porto, Portugal \\ ${ }^{3}$ Department of Pathology, Faculty of Medicine of the University of Porto (FMUP), Porto, Portugal \\ ${ }^{4}$ Institute of Molecular Pathology and Immunology at the University of Porto (Ipatimup), Instituto de Investigação e Inovação em Saúde (i3S), Porto, Portugal
}

\begin{abstract}
Animal-type melanoma, also referred as pigmented epithelioid melanocytoma, is an unusual melanocytic tumor with striking pigment synthesis. Although many patients may develop lymph-node or even distant metastases, animal-type melanoma show a more indolent clinical course than conventional melanoma. The recognition of uncommon melanocytic lesions, such as animal-type melanoma, is pivotal to guarantee the proper diagnosis and the consequent correct management of the lesion. Herein, we report the case of a pigmented lesion of the scalp, whose pathological diagnosis of animal-type melanoma guaranteed the correct clinical management. We therefore discuss the main differential diagnoses from the clinical and pathological point of view.
\end{abstract}

\section{Introduction}

Animal-type melanoma, also referred as pigmented epithelioid melanocytoma, is an unusual melanocytic tumor with striking pigment synthesis. Few cases of animal-type melanoma have been reported [1].

We present a case of a pigmented lesion of the scalp whose pathological diagnosis of animal-type melanoma guaranteed the correct clinical management.

\section{Case Description}

A 82 -year-old caucasian man was observed with a $50 \mathrm{~mm}$ brown, nodular and exophytic lesion of the scalp, with two years of evolution. Wide excision (Figure 1) and plasty with split-thickness skin graft was performed (Figure 2). Histology revealed a heavily pigmented wellcircumscribed tumour, extending to the dermal subcutaneous interface, with epidermal ulceration, determining the effacement of normal dermal structures. The tumour presented a nodular architecture, was composed of large epithelioid to spindled cells, often containing

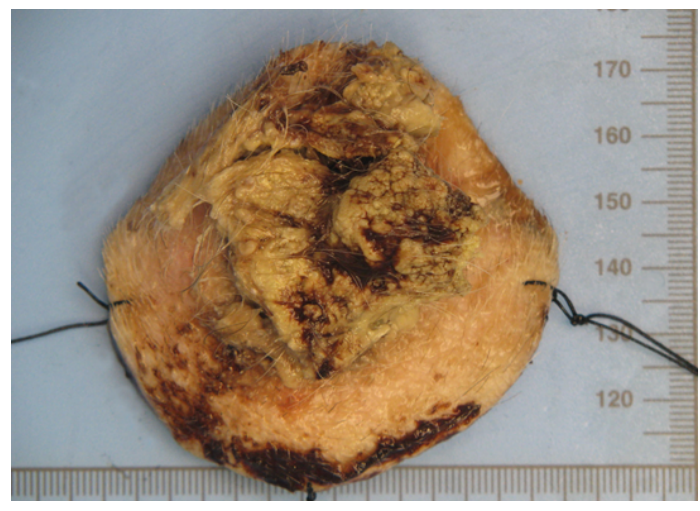

Figure 1. Surgical resection specimen. cytoplasmic melanic pigment, with nuclear atypia, pleomorphism and prominent nucleoli. Aggregates of melanophages were seen throughout the lesion. Mitotic figures were observed $\left(3 / \mathrm{mm}^{2}\right)$. No melanoma in situ component was observed. Immunohistochemical study showed diffuse immunoreactivity for S100, HMB-45 and MelanA in the neoplastic cells, confirming the pathological diagnosis of animal-type melanoma (Figure 2).

Cervical and thoraco-abdomino-pelvic CT scan didn't reveal local or distant metastasis. Biopsy of sentinel lymph node was proposed, but the patient refused. After 2 years the patient is disease free, with no recurrent lesions and completely healed (Figure 3).

\section{Discussion}

We report a rare case of an elderly male with animal type melanoma, a rare melanocytic tumour with distinct clinical and histopathological findings that is more common in a younger population [1].

The first interesting fact is that animal-type melanoma is more common in a younger population (people with less than 30 years most commonly affected) [1], so it was not expected in an 82-year-old man.

Animal-type melanoma lesions are classically blackened nodules or plaques measuring about $1 \mathrm{~cm}$ in diameter, up to $10 \mathrm{~cm}$, with long time evolution, being characterized for its indolent behavior [1]. Our patient presented with a brown exophytic lesion, different from

${ }^{\star}$ Correspondence to: Barreiro D, Department of Plastic Surgery and Burn Unit, Centro Hospitalar São João, Alameda Prof. Hernâni Monteiro 4200-319, Porto, Portugal, Tel: +351 225512 100; E-mail: diogobarreiro@hotmail.com

Key words: animal-type melanoma, pigment-synthesizing melanoma, pigmented epithelioid melanocytoma

Received: July 20, 2020; Accepted: August 13, 2020; Published: August 17, 2020 


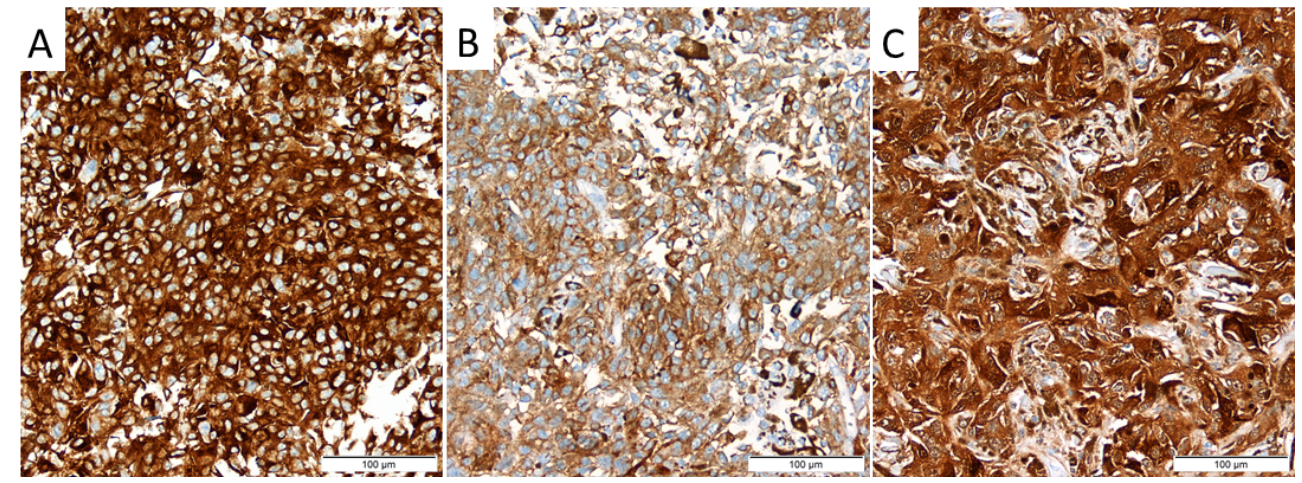

Figure 2. Immunohistochemical study showed immunoreactivity in the neoplastic cells of (A) Melan A, (B) HMB45 and (C) S100

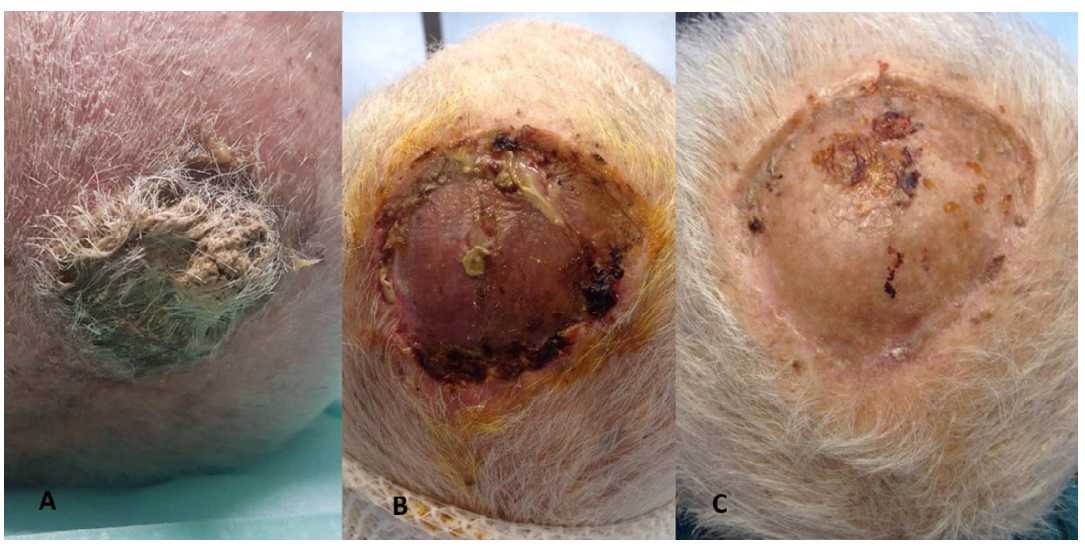

Figure 3. (A) Skin lesion before resection. (B) 2 weeks after wide excision and plasty with split-thickness skin graft. (C) 2 years after wide excision and plasty with split-thickness skin graft

the blackened lesions described, so macroscopically it appointed to squamous cell carcinoma, basal cell carcinoma, the rare Merkel cell carcinoma or even atypical fibroxanthoma. For our surprise, histology and immunohistochemical study made the diagnosis of animal-type melanoma.

Animal-type melanoma occurs equally in men and women, with caucasians most commonly affected [2]. The median Breslow depth is $3.8 \mathrm{~mm}^{2}$, ulceration is reported in few cases and dermal mitoses $\geq 1 / \mathrm{mm}^{2}$ is reported in $27.4 \%$ [2]. Patients that underwent sentinel lymph node biopsy had $41.0 \%$ positivity rate [2]. Those who underwent lymph node dissection had $34.4 \%$ positivity rate [2]. In a study with 190 patients, loco-regional recurrence was reported in 15 patients, recurrence with distant metastases in 6 patients, and death in 5 patients [2].

There is no relationship with familial dysplastic nevus syndrome, sun exposure, Carney complex or family history of melanoma [1].

Differential diagnoses of animal type melanoma include [3,4]: (a) epithelioid blue nevus which encompasses the detection of a typical blue nevus component with organoid configuration, dendritic melanocytes, and non-scarring fibrosis; (b) nodular melanosis with striking melanic pigment has a potential relationship to animal-type melanomas even more for its content of melanophages, and possible presence of melanin-laden melanoma cells in the two entities; (c) largely regressed melanoma with a prominent accumulation of melanophages, which has a characteristic pattern of delicate fibrosis in the middle of the edematous matrix containing scarce mononuclear cells and prominent venules; (d) heavily melaninized metastatic melanoma may potentially also be indistinguishable from animal-type melanoma, and clinical information may be necessary for distinction of the two entities; (e) cellular blue nevi including atypical and malignant variants are commonly biphasic lesions with two components: a paucicellular pigmented dendriticsclerotic component and an amelanotic component comprised of bundles of spindled melanocytes; (f) malignant blue nevus typically has, in depth, a melanocytic proliferation consistent with cellular blue nevus, generally located laterally to the malignant component.

As found in our patient's lesion, animal-type melanoma cells demonstrate positivity for S-100 protein, vimentin, HMB-45 and Melan-A [3,4], confirming the melanocytic nature of the lesion.

Few cases of animal type melanoma have been described [1]. The biological behaviour is uncertain. Patients appear to manifest a long, indolent phase with few cases having recurrence after one year of surgical excision. One-third may have systemic spread: metastases to the spleen, liver, bone marrow and parotid have been described. Nevertheless, patients rarely die of the disease [1,5]. In most cases, the evolution is less aggressive than in nodular or superficial spreading melanomas in the vertical growth phase with similar histological parameters [1]. Although generally considered a neoplasm with an indolent course, animal type melanoma may have an aggressive behavior as it can recur locally and spread to regional lymph nodes $[1,6,7]$. Distant metastases are relatively rare and, when they occur, patients appear to have a better prognosis than patients with conventional melanoma [2,5], despite several authors reported patients with animal-type melanoma who died of melanoma metastases [5,8]. Ludgate et al. [6] reported that no patients with negative sentinel nodes developed recurrent disease. 
The recommended treatment is complete excision of the lesion with 1-2 $\mathrm{cm}$ margins and sentinel node biopsy $[1,2,6]$.

\section{Conclusions}

Animal type melanoma is a rare melanocytic neoplasm, with indolent evolution, despite the occurrence of lymph node and distant metastasis. The histopathological differential diagnoses included melanocytic lesions with striking pigment synthesis. It has uncertain biological behavior and apparently have better prognosis than other types of melanoma. The rarity of animal type melanoma leads to scarce knowledge of the biological behavior of this particular neoplasm. Further studies and longer follow-up data are needed to better define the prognosis and the staging, therapeutic and follow-up strategies for the patients.

\section{Conflict of interest}

There are no conflicts of interest to disclose for this study for any of the authors listed.

\section{References}

1. Magro CM, Crowson AN, Mihm MC (2006) Unusual variants of malignant melanoma. Mod Pathol 19: S41-S70. [Crossref]

2. Vyas R, Keller JJ, Honda K, Cooper KD, Gerstenblith MR (2015) A systematic review and meta-analysis of animal-type melanoma. J Am Acad Dermatol 73: 1031-1039. [Crossref]

3. Patterson, J., (2016) Weedon's skin pathology. 4th ed. The Netherlands: Elsevier Health Sciences pp.898-899.

4. Barnhill, R., Piepkorn M.W., Busam K.J., (2014) Pathology of Melanocytic Nevi and Melanoma. 3rd ed. Berlin: Springer Science \& Business Media pp.445-449

5. Vezzoni GM, Martini L, Ricci C (2008) A Case of Animal-Type Melanoma (or Pigmented Epithelioid Melanocytoma?): An Open Prognosis. Dermatologic Surg 34: 105-109. [Crossref]

6. Ludgate MW, Fullen DR, Lee J, Rees R, Sabel MS, et al. (2010) Animal-type melanoma: A clinical and histopathological study of 22 cases from a single institution. Br J Dermatol 162: 129-136. [Crossref]

7. Sestini S, Gerlini G, Brandani P, Gelli R, Talini G, et al. (2012) “Animal-type” melanoma of the scalp with satellitosis and positive sentinel nodes in a 4-year-old child: Case report and review of the literature. J Plast Reconstr Aesthetic Surg 65: e90-e94. [Crossref]

8. Crowson AN, Magro CM, Mihm MC (1999) Malignant melanoma with prominent pigment synthesis: "Animal type" melanoma - A clinical and histological study of six cases with a consideration of other melanocytic neoplasms with prominent pigment synthesis. Hum Pathol 30: 543-550. [Crossref]

Copyright: (C2020 Barreiro D. This is an open-access article distributed under the terms of the Creative Commons Attribution License, which permits unrestricted use, distribution, and reproduction in any medium, provided the original author and source are credited. 